\title{
Minimally Invasive Sacroiliac Joint Fusion: One-Year Outcomes in 40 Patients
}

\author{
Donald Sachs ${ }^{1}$ and Robyn Capobianco ${ }^{2}$ \\ ${ }^{1}$ Center for Spinal Stenosis and Neurologic Care, P.O. Box 8815, Lakeland, FL 33806, USA \\ ${ }^{2}$ SI-BONE Inc., 3055 Olin Ave. Suite 2200, San Jose, CA 95128, USA \\ Correspondence should be addressed to Robyn Capobianco; spinewriter@gmail.com
}

Received 4 June 2013; Revised 16 July 2013; Accepted 17 July 2013

Academic Editor: Panagiotis Korovessis

Copyright ( 2013 D. Sachs and R. Capobianco. This is an open access article distributed under the Creative Commons Attribution License, which permits unrestricted use, distribution, and reproduction in any medium, provided the original work is properly cited.

\begin{abstract}
Background. SI joint pain is difficult to diagnose due to overlapping symptoms of the lumbar spine, and until recently, treatment options have been limited. The purpose of this retrospective study is to report on the safety and effectiveness of MIS SI joint arthrodesis using a series of triangular, porous plasma coated implants in patients refractory to conservative care. Methods. We report on the first 40 consecutive patients with one-year follow-up data that underwent MIS SI joint fusion with the iFuse Implant System (SI-BONE, Inc., San Jose, CA) by a single surgeon. Medical charts were reviewed for demographics, perioperative metrics, complications, pain scores, and satisfaction. Results. Mean age was 58 years (range 30-81) and 75\% of patients were female. Postoperative complications were minimal and included transient trochanteric bursitis (5\%), facet joint pain (20\%), and new low back pain (2.5\%). There were no reoperations at one year. Mean pain score improved from 8.7 (1.5 SD) at baseline to 0.9 (1.6) at 12 months, a 7.8-point improvement $(P<.001)$. Patient satisfaction was very high. Conclusions. The results of this case series reveal that MIS SI joint fusion using the iFuse Implant System is a safe and effective treatment option in carefully selected patients.
\end{abstract}

\section{Background}

Low back pain (LBP) is exceedingly common in modern society, affecting well over $90 \%$ of adults at some point in their lives [1]. Apart from the common cold, it is the most common reason for visits to the primary care doctor [1]. Loss of productivity and income combined with medical expenses results in a $\$ 60$ billion expenditure annually in the US related to low back pain [2]. Successful treatment of low back pain demands identifying the pain generator(s), which can be a significant challenge due to the multifactorial nature of this condition. In the early 1900s, the sacroiliac (SI) joint was suspected as a significant generator of LBP. Over time, as more reliably diagnosed conditions such as herniated discs and facet arthropathy became better understood, less focus was placed on the SI joint [3]. Recently there has been a resurgence in consideration of the SI joint as a low back pain generator. Recent published literature reports that $15-30 \%$ of patients presenting with low back pain had SI joint problems [4]. Additionally, up to $75 \%$ of postlumbar fusion patients will develop significant SI joint degeneration after 5 years [57]. SI joint pain can mimic discogenic or radicular low back pain, and patients can present with low back, groin, and/or gluteal pain, leading to the potential for inaccurate diagnosis and treatment $[1,8,9]$.

Despite the large number of patients with SI joint pain, treatment options have been limited to conservative care involving physical therapy and joint injections or traditional open SI joint arthrodesis surgery until recently. Open arthrodesis procedures reported in the literature require relatively large incisions, significant bone harvesting, and lengthy hospital stays; moreover, they may require nonweight bearing for several months [10-13].

Recent case series reports of a minimally invasive arthrodesis system (iFuse Implant System, SI-BONE Inc., San Jose, CA) have shown excellent outcomes [14-17]. The surgical procedure involves placing a series of triangular, porous plasma spray coated titanium implants placed across the SI joint without the use of second site bone harvesting or 
graft. We wished to determine if the single center outcomes in the literature were commensurate with our own. The purpose of this retrospective study is to report on the safety and effectiveness of this procedure in a single surgeon's private practice.

\section{Methods}

We report outcomes of the first consecutive 40 patients with one-year follow-up data treated at a single, community-based spine practice between April 2011 and March 2012. Medical charts were reviewed for perioperative metrics, complications, and pain scores using a numerical rating scale (NRS) preoperatively and at 6 weeks, 3-, 6-, and 12-months postoperatively. Patient satisfaction with surgical results (yes or no) was obtained at 12 months post-operatively. IRB approval was obtained before beginning the study.

Mean age was 58 years (range $30-81$ ), and three quarters of the patients were women $(75 \%)$ (Table 1). Patients were diagnosed with either degenerative sacroiliitis or sacroiliac joint disruption using a combination of history, clinical exam, and positive diagnostic injection. Degenerative sacroiliitis is defined as a degeneration of the joint, either osteoarthritic or as a result of adjacent segment disease after fusion. Sacroiliac joint disruption is a physical separation of the joint, typically as a result of trauma. Patients presented with SI joint pain and all but one complained of low back pain. Additional symptoms were buttock pain (60\%) and groin pain $(13 \%)$. Nearly half $(48 \%)$ had a history of previous lumbar spine surgery that included: fusion at one or more levels $(63 \%)$, decompression (16\%), discectomy (10.5\%), and $10.5 \%$ with nonspecific documented procedures.

All patients failed a minimum 6-month course of conservative care consisting of medication optimization, physical therapy, and SI joint injections. A thorough physical and clinical exam was performed on all patients in order to determine the primary pain generator as accurately as possible in this complex back pain population. Positive results on 3 or more provocative physical examination maneuvers (such as FABER, compression, thigh thrust, distraction, and Gaenslen) were used as criteria to guide subsequent diagnostic activities [18]. Diagnostic imaging studies (MRI and/or CT scan) were performed to assess pathology in the lumbopelvic hip complex. When clinical, physical, and imaging examinations were concordant, patients were sent for confirmatory image-guided diagnostic injections of the SI joint using long acting anesthetic. A 75\% reduction in pain immediately following injection of local anesthetic was used to confirm the SI joint as a pain generator [7].

Minimally invasive SI joint fusion using the iFuse Implant System (SI-BONE Inc., San Jose, CA) was performed in all cases by a single neurosurgeon in private practice. This system entails the placement of 3 triangular, porous plasma coated titanium implants across the SI joint in order to stabilize and fuse the joint without the need for additional bone graft.

2.1. Technique Overview. The procedure is performed with the patient positioned prone on a radiolucent table and under
TABle 1: Patient demographics.

\begin{tabular}{ll}
\hline Patients & 40 \\
Age & 58 (range 30-81) \\
Gender & $30 \mathrm{~F}(75 \%), 10 \mathrm{M}(25 \%)$ \\
& $39(98 \%)$ LBP \\
Symptoms & $24(60 \%)$ buttock pain \\
& $5(13 \%)$ groin pain \\
& $19(48 \%)$ total \\
Prior lumbar spine & 12 fusion, 3 decompression, 1 unknown, \\
surgery & 2 discectomy, 1 spinal cord stimulator \\
\hline
\end{tabular}

general endotracheal anesthesia. Intermittent fluoroscopy is used to monitor instrument and implant placement. After a $3 \mathrm{~cm}$ lateral incision is made into the buttock region, the gluteal fascia is bluntly dissected to reach the outer table of the ilium. A Steinmann pin is passed through the ilium across the SI joint into the center of the sacrum, lateral to the neural foramen. A soft tissue protector is inserted over the pin, and a drill is used to create a pathway and decorticate bone through the ilium to the sacrum. After the drill is removed, a triangular broach is used before the first implant is malleted into place. A total of three implants are placed. (Figure 1). In some cases more than three implants can be used, but in this series all patients had three implants. The most cephalad implant is seated within the sacral ala. A pin-guide system is used to facilitate placement of the subsequent implants. The second implant is generally located above or adjacent to the S1 foramen and the third between the S1 and S2 foramens. The incision is then irrigated, and the tissue layers are closed with Vicryl and Monocryl sutures. Patients are instructed to ambulate with the assistance of a walker for the first 4 weeks after which time toe touch ambulation is recommended for another 4 weeks. After patients have undergone this 8-week program of a gradual return to full weight bearing, they begin 4 weeks of physical therapy.

2.2. Outcomes. Pain related to the SI joint was assessed preoperatively and postoperatively at 12 months. Patients were asked to rate their pain using a $0-10$ numerical rating scale with 0 representing no pain and 10 representing the worst pain imaginable. Satisfaction was assessed by asking the patient (yes or no) if s/he would have the same surgery again for the same outcome.

\section{Results}

A total of 41 SI joints in 40 patients were treated: 17 right- and 24 left sided. One patient underwent bilateral surgery (Table 2). One patient underwent concomitant L3/4 laminectomy, foraminotomy, and facetectomy. Blood loss was minimal $(<50 \mathrm{cc})$ in all cases, and most patients are kept in the hospital overnight. Surgery time was not available for all patients. We previously reported an operating time of $78 \pm 32$ minutes in a subset of this cohort [15]. No intraoperative 


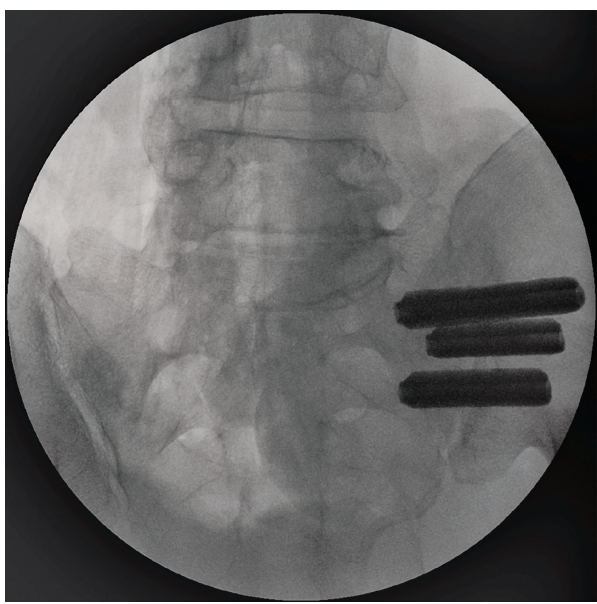

(a)

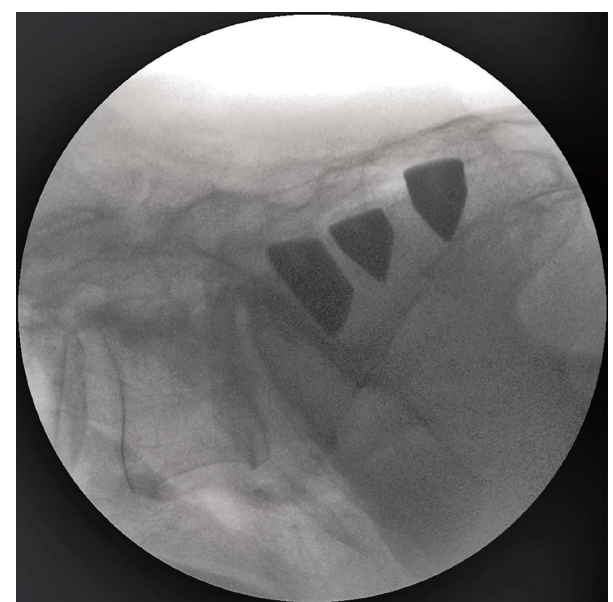

(b)

FIgURE 1: (a) AP and (b) lateral view of all three implants in place.

complications were observed. At one year, there were no surgical revisions.

3.1. Clinical Outcomes. Mean $( \pm \mathrm{SD})$ preoperative pain score as measured using a numerical rating scale (NRS) was $8.7 \pm$ 1.5. Improvement in pain was observed as early as the 6-week follow-up visit (mean $1.2 \pm 1.7$ ), and patients continued to have symptom relief at the 3- and 6-month follow-up visits, means $0.6 \pm 1.2$ and $0.8 \pm 1.8$, respectively. This improvement was durable through the 12-month followup with a reported mean pain score of $0.9( \pm 1.6)$. The mean $( \pm S D)$ change in pain score was $-7.8( \pm 2.3)$ points $(t$ test, $P<.001)$. A subgroup analysis revealed that there was no difference in outcomes between patients with and without prior lumbar spinal fusion. A clinically significant benefit, defined as a $>2$ point change from baseline, was observed in all but one patient [19]. Patient satisfaction was extremely high with all patients (100\%) indicating that they would have the same surgery again for the same result.

\subsection{Complications. There were no intraoperative complica-} tions. Two patients presented with trochanteric bursitis, 1 incident of piriformis syndrome, and 1 episode of new low back pain (Table 3). Eight patients continued to have facet pain, which was present preoperatively. During the postoperative follow-up period, 2 patients with preexisting lower back pain due to degenerative disc disease and severe spinal stenosis underwent lumbar fusion. One patient underwent discectomy at L4/5. All three surgeries were unrelated to the index procedure.

\section{Discussion}

SI joint symptoms can present as pain in the SI joint, low back, hip, groin, or buttock. As a result, a careful and thorough clinical and physical exam must be performed to correctly identify the pain generator(s). Positive provocative physical
TABle 2: Perioperative characteristics.

\begin{tabular}{ll}
\hline Joints treated & 41 \\
Right SI joint & 17 \\
Left SI joint & 24 \\
Concomitant spine & $\begin{array}{l}\text { 1: L3/4 laminectomy, facetectomy, } \\
\text { procedures }\end{array}$ \\
\hline
\end{tabular}

TABle 3: Postoperative complications and events.

\begin{tabular}{ll}
\hline Piriformis syndrome & 1 \\
New low back pain & 1 \\
Facet joint pain & 8 \\
Trochanteric bursitis & 2 \\
Discectomy at L4/5 & 1 \\
Lumbar spine fusion & 1 case at L2/3 due to severe spinal stenosis \\
& 1 case at L3/4 for degenerative disc disease \\
\hline
\end{tabular}

examination maneuvers (such as FABER, Gaenslen, and Thigh Thrust) combined with marked (e.g., $75 \%$ or greater) pain relief after image-guided SI joint injection are a reliable method for diagnosing the SI joint as a pain generator $[7,18$, 20].

Recent reports of other MIS approaches to SI joint arthrodesis using screws show relatively good clinical results with room for improvement in outcomes and technique [16]. Al-Khayer et al. reported on 9 patients using a single hollow modular anchorage (HMA) screw packed with bone graft [21]. All patients experienced a clinically significant improvement in VAS pain scores, and all but 1 patient improved in function as measured by ODI. One patient suffered a deep wound infection. Khurana et al. also report on HMA screws with demineralized bone matrix in a cohort of 15 patients with relatively good outcomes [22]. Wise and 
Dall reported on 13 patients and 19 joints using $11 \times 25 \mathrm{~mm}$ threaded fusion cages packed with rhBMP-2 with good clinical results [3]. Both of these MIS techniques, which use rhBMP-2 or autologous bone graft, have substantial drawbacks. The use of rhBMP-2 has come under fire for unreported adverse events as well as unapproved uses [23], and autologous iliac crest harvesting can lead to further degeneration of the SI joint [10]. Additionally, the use of cages and screws for SI joint fusion may not be appropriate for patients with a history of instrumented spinal surgery. Mason et al. reported significantly worse outcomes after SI joint fusion using HMA screws in patients with a history of previous lumbar spine surgery [24]. Further studies are needed to assess the incidence of screw loosening, breakage, and need for hardware removal as these events have been reported in association with other spine procedures using orthopedic screws [19].

Several case series reports using the same MIS technique used in this current study report favorable results with minimal complications and no suggestion of implant loosening $[14,15,17]$. In a case series of 50 patients, the author reported clinically and statistically significant improvements in pain and function independent of a prior history of lumbar spine fusion [16]. Similarly, there was no difference in outcomes between patients with and without history of lumbar spinal fusion in our study.

Advantages of MIS SI joint fusion using the iFuse Implant System include a small incision, relatively short operating time, minimal blood loss, a relatively short period of immobilization, and most importantly bone and ligament preservation. The triangular shape combined with an interference fit of the titanium implant used in this cohort was designed to minimize rotation, and micromotion and avoid issues encountered with traditional screws. In our cohort of patients undergoing MIS SI joint fusion, clinical outcomes were favorable with $98 \%$ of patients experiencing a clinically significant benefit at 12 months.

Postoperative complications were minimal. Two patients (5\%) went on to have fusion surgery for significant degenerative disc disease or spinal stenosis. Both conditions were present prior to SI joint fusion surgery; however the patient's chief complaint was the SI joint necessitating primary attention to this area. There were 2 cases (5\%) of transient trochanteric bursitis and 1 episode of piriformis syndrome. These are neither uncommon nor unexpected and can be a result of altered gait pattern due to low back or hip pain, postoperative hip abductor weakness, and other trauma in the region [19]. Facet pain was present in $20 \%$ of our patients, but it is unclear whether these patients had symptoms prior to the surgery. These patients were treated with either facet injections or physical therapy, depending on severity and patient preference.

Although our study sample size is small, the results of minimally invasive SI joint surgery appear promising. All patients presented with low back and SI joint pain. Favorable outcomes in these patients underscore the necessity to suspect the SI joint as a pain generator in patients with low back pain. Special attention should be paid to the SI joint after lumbar spine surgery to avoid the potential for inaccurate diagnosis and treatment. Furthermore, this minimally invasive approach may significantly benefit the elderly population, who are not candidates for other conventional techniques due to poor bone quality, delayed healing and reduced mobility. Thirty-eight percent (38\%) of our patients were over the age of 65 . This segment of the population is not likely to respond well to physical therapy alone in part because of the degenerative nature of SI joint disease. The MIS procedure described herein may afford this segment of the population an opportunity to regain mobility, alleviate SI joint and low back pain caused by SI joint issues, and experience an improved quality of life.

\section{Conclusion}

When conservative measures fail, minimally invasive SI joint fusion using a series of triangular porous plasma coated titanium implants is a safe and effective treatment option in carefully selected patients. Additional prospective controlled trials are underway.

\section{List of Abbreviations}

SI: Sacroiliac

NRS: Numerical rating scale

MIS: Minimally invasive surgery

LBP: Low back pain.

\section{Competing Interests}

No funds were received for this research. Donald Sachs is a paid consultant for SI-BONE Inc. Robyn Capobianco is an employee of SI-BONE Inc.

\section{Authors' Contributions}

Donald Sachs performed all surgeries, assisted in paper draft, and reviewed final paper. Robyn Capobianco prepared study protocol, IRB documentation, performed data analysis, and drafted paper.

\section{Authors' Information}

Donald Sachs is a board certified neurosurgeon specializing in minimally invasive spine surgery. Robyn Capobianco is a clinical research professional and medical writer.

\section{Acknowledgments}

The authors wish to acknowledge Daniel Cher, MD for data analysis and critical editorial review, Deidre Sachs, and Kristen Ball for data acquisition.

\section{References}

[1] N. Weksler, G. J. Velan, M. Semionov et al., "The role of sacroiliac joint dysfunction in the genesis of low back pain: 
the obvious is not always right," Archives of Orthopaedic and Trauma Surgery, vol. 127, no. 10, pp. 885-888, 2007.

[2] W. Murray, "Sacroiliac joint dysfunction: a case study," Orthopaedic Nursing, vol. 30, no. 2, pp. 126-131, 2011.

[3] C. L. Wise and B. E. Dall, "Minimally invasive sacroiliac arthrodesis: outcomes of a new technique," Journal of Spinal Disorders and Techniques, vol. 21, no. 8, pp. 579-584, 2008.

[4] J. N. Sembrano and D. W. Polly, "How often is low back pain not coming from the back?" Spine, vol. 34, no. 1, pp. E27-E32, 2009.

[5] K.-Y. Ha, J.-S. Lee, and K.-W. Kim, "Degeneration of sacroiliac joint after instrumented lumbar or lumbosacral fusion: a prospective cohort study over five-year follow-up," Spine, vol. 33, no. 11, pp. 1192-1198, 2008.

[6] M. J. Depalma, J. M. Ketchum, and T. R. Saullo, "Etiology of chronic low back pain in patients having undergone lumbar fusion," Pain Medicine, vol. 12, no. 5, pp. 732-739, 2011.

[7] J. Y. Maigne, A. Aivaliklis, and F. Pfefer, "Results of sacroiliac joint double block and value of sacroiliac pain provocation tests in 54 patients with low back pain," Spine, vol. 21, no. 16, pp. 18891892, 1996.

[8] B. S. Foley and R. M. Buschbacher, "Sacroiliac joint pain: anatomy, biomechanics, diagnosis, and treatment," American Journal of Physical Medicine and Rehabilitation, vol. 85, no. 12, pp. 997-1006, 2006.

[9] A. C. Schwarzer, C. N. Aprill, and N. Bogduk, "The sacroiliac joint in chronic low back pain," Spine, vol. 20, no. 1, pp. 31-37, 1995.

[10] J. M. Buchowski, K. M. Kebaish, V. Sinkov, D. B. Cohen, A. N. Sieber, and J. P. Kostuik, "Functional and radiographic outcome of sacroiliac arthrodesis for the disorders of the sacroiliac joint," The Spine Journal, vol. 5, no. 5, pp. 520-528, 2005.

[11] K. A. Giannikas, A. M. Khan, M. T. Karski, and H. A. Maxwell, "Sacroiliac joint fusion for chronic pain: a simple technique avoiding the use of metalwork," European Spine Journal, vol. 13, no. 3, pp. 253-256, 2004.

[12] M. N. Smith-Petersen, "Arthrodesis of the sacroiliac joint. A new method of approach," The Journal of Bone \& Joint Surgery, vol. 3, no. 8, pp. 400-405, 1921.

[13] M. R. Moore, "Surgical treatment of chronic painful sacroiliac joint dysfunction.", in Movement, Stability, and Low Back Pain: The Essential Role of the Pelvis, pp. 563-572, Churchill Livingstone, New York, NY, USA, 1997.

[14] L. Rudolf, "Sacroiliac joint srthrodesis-MIS technique with titanium implants: report of the first 50 patients and outcomes," The Open Orthopaedics Journal, vol. 6, no. 1, pp. 495-502, 2012.

[15] D. Sachs and R. Capobianco, "One year successful outcomes for novel sacroiliac joint arthrodesis system," Annals of Surgical Innovation and Research, vol. 6, no. 1, article 13, 2012.

[16] L. Rudolf, "MIS fusion of the SI Joint: does prior lumbar spinal fusion affect patient outcomes?" The Open Orthopaedics Journal, vol. 7, pp. 163-168, 2013.

[17] J. T. Kim, L. M. Rudolf, and J. A. Glaser, "Outcome of percutaneous sacroiliac joint fixation with porous plasma-coated triangular titanium implants: an independent review.," The Open Orthopaedics Journal, vol. 7, pp. 51-56, 2013.

[18] K. M. Szadek, P. van der Wurff, M. W. van Tulder, W. W. Zuurmond, and R. S. G. M. Perez, "Diagnostic validity of criteria for sacroiliac joint pain: a systematic review," Journal of Pain, vol. 10, no. 4, pp. 354-368, 2009.

[19] A. G. Copay, S. D. Glassman, B. R. Subach, S. Berven, T. C. Schuler, and L. Y. Carreon, "Minimum clinically important difference in lumbar spine surgery patients: a choice of methods using the Oswestry Disability Index, Medical Outcomes Study questionnaire Short Form 36, and Pain Scales," The Spine Journal, vol. 8, no. 6, pp. 968-974, 2008.

[20] P.-C. Liliang, K. Lu, C.-L. Liang, Y.-D. Tsai, K.-W. Wang, and H.-J. Chen, "Sacroiliac joint pain after lumbar and lumbosacral fusion: findings using dual sacroiliac joint blocks," Pain Medicine, vol. 12, no. 4, pp. 565-570, 2011.

[21] A. Al-Khayer, J. Hegarty, D. Hahn, and M. P. Grevitt, "Percutaneous sacroiliac joint arthrodesis: a novel technique," Journal of Spinal Disorders and Techniques, vol. 21, no. 5, pp. 359-363, 2008.

[22] A. Khurana, A. R. Guha, K. Mohanty, and S. Ahuja, "Percutaneous fusion of the sacroiliac joint with hollow modular anchorage screws: clinical and radiological outcome," Journal of Bone and Joint Surgery B, vol. 91, no. 5, pp. 627-631, 2009.

[23] E. J. Carragee, A. J. Ghanayem, B. K. Weiner, D. J. Rothman, and C. M. Bono, "A challenge to integrity in spine publications: years of living dangerously with the promotion of bone growth factors," The Spine Journal, vol. 11, no. 6, pp. 463-468, 2011.

[24] L. W. Mason, I. Chopra, and K. Mohanty, "The percutaneous stabilisation of the sacroiliac joint with hollow modular anchorage screws: a prospective outcome study," European Spine Journal, 2013. 


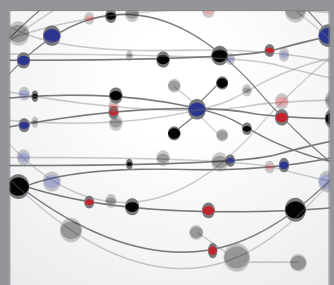

The Scientific World Journal
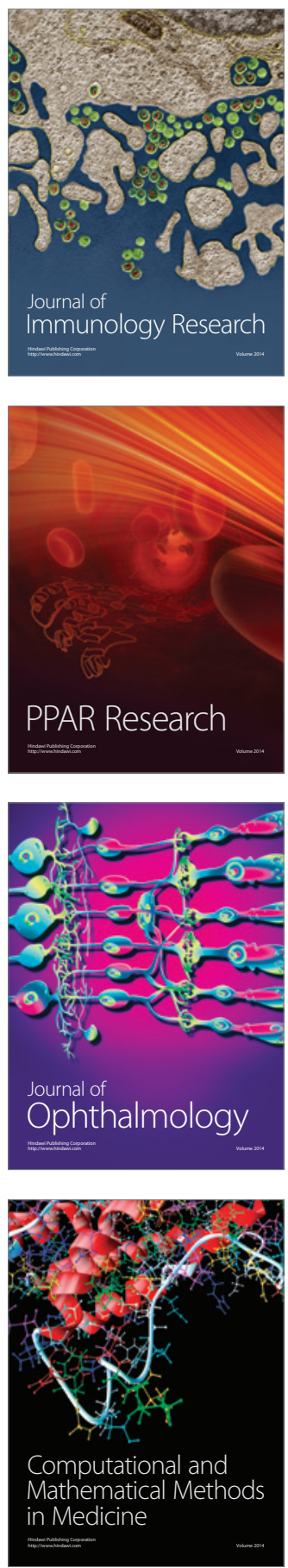

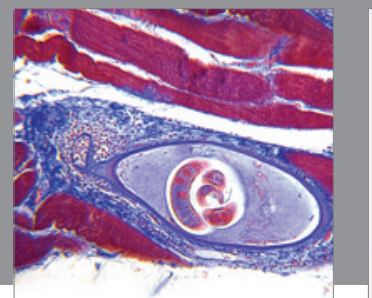

Gastroenterology

Research and Practice
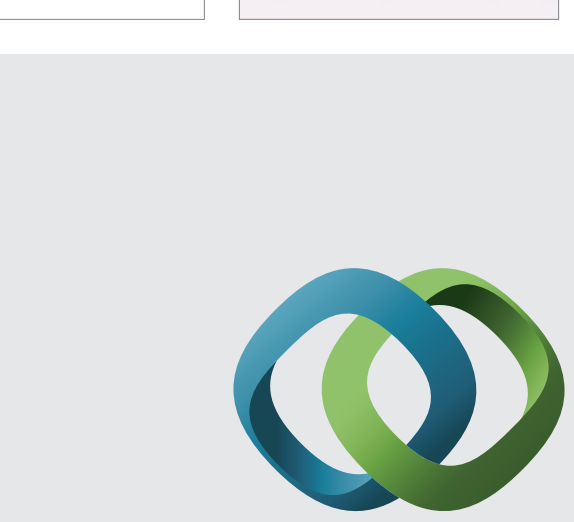

\section{Hindawi}

Submit your manuscripts at

http://www.hindawi.com
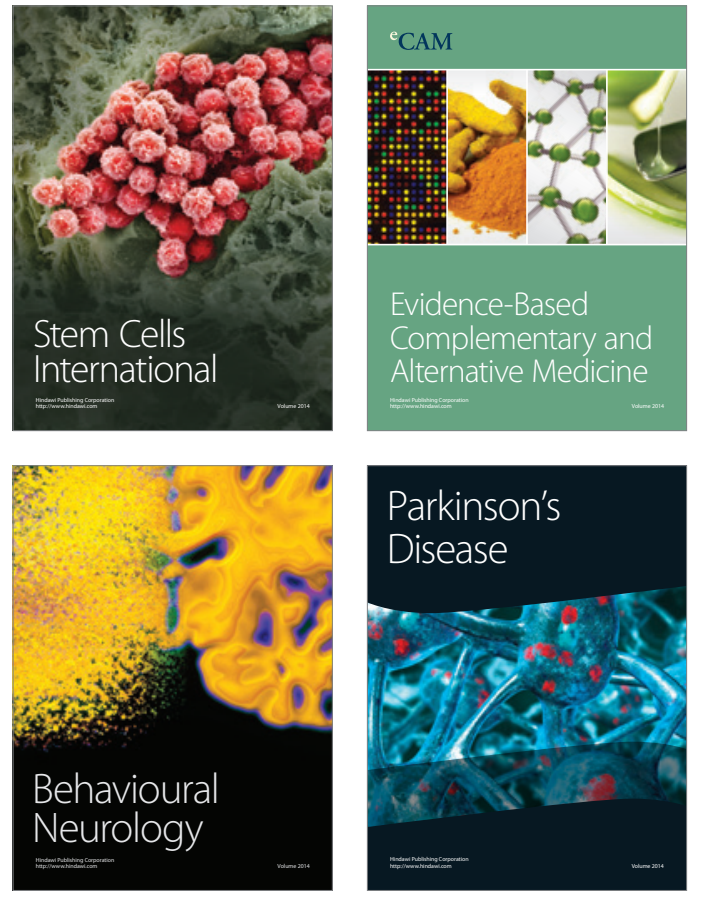
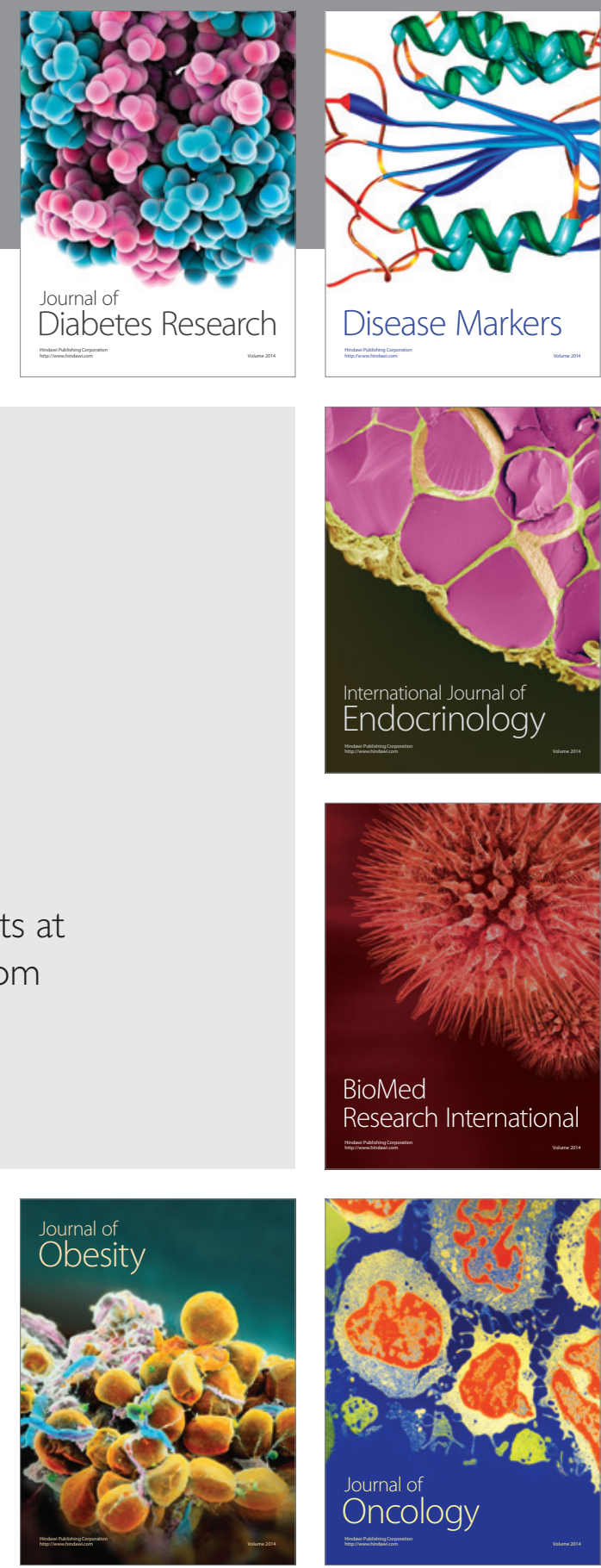

Disease Markers
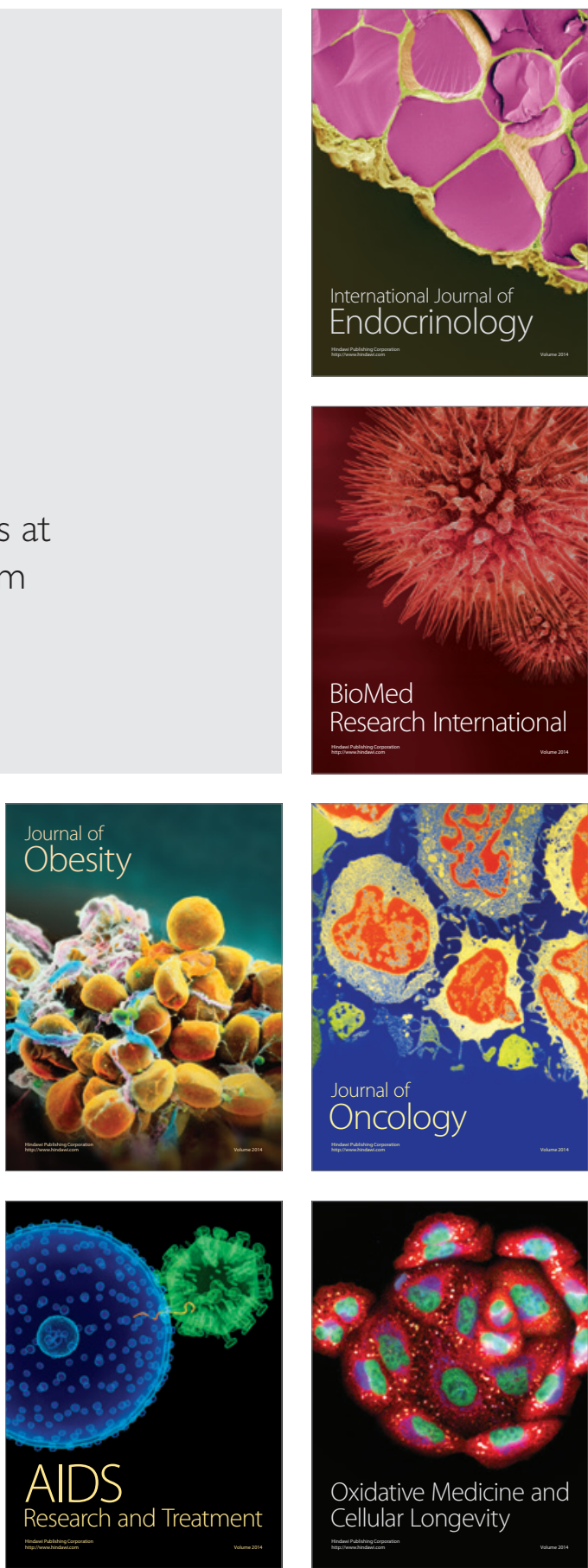